\title{
RECAPTURING A HOLOMORPHIC FUNCTION ON AN ANNULUS FROM ITS MEAN BOUNDARY VALUES
}

\author{
CHIN-HUNG CHING AND CHARLES K. CHUI
}

\begin{abstract}
Let $D$ be an annulus in the complex plane with closure $\bar{D}$ and boundary $\partial D$. We prove that a function $f$, holomorphic in $D$ with $C^{1+\varepsilon}(\partial D)$ boundary data for some $\varepsilon>0$, is uniquely determined by its arithmetic means $s_{n}(f)$ and $s_{0 n}(f)$ over equally spaced points on $\partial D$. We also give an explicit formula for recapturing $f$ from its means $s_{n}(f)$ and $s_{0 n}(f)$. Furthermore, we derive the relations between $s_{n}(f)$ and $s_{0 n}(f)$ which are necessary and sufficient for the analytic continuability of $f$ from $D$ to the whole disc.
\end{abstract}

1. Introduction. Let $U:|z|<1$ be the open unit disc and $T:|z|=1$ be the unit circle in the complex plane. For an $\varepsilon>0$, we let $A^{1+\varepsilon}(U)$ denote the class of all functions

$$
f(z)=\sum_{n=0}^{\infty} a_{n} z^{n}
$$

with $a_{n}=O\left(1 / n^{1+\varepsilon}\right)$. If $f$ is a continuous function on $T$, we consider the arithmetic means

$$
s_{n}(f)=\frac{1}{n} \sum_{k=1}^{n} f\left(w_{n}^{k}\right),
$$

$n=1,2, \cdots \cdot$, of $f$ on $T$, where $w_{n}^{k}=\exp (i 2 \pi k / n)$ are the $n$th roots of unity. It is known (cf. [1]) that if $f \in A^{1+\varepsilon}(U)$ then the sequence $\left\{s_{n}(f)\right\}$ uniquely determines $f$ in $A^{1+\varepsilon}(U)$. Also, an explicit representation of a function $f$ in $A^{1+\varepsilon}(U)$ in terms of the sequence $\left\{s_{n}(f)\right\}$ is given in [3]. In this paper, we establish these results for functions holomorphic in an annulus. Hence, one can explicitly recapture a function $f$, holomorphic in a simply connected or doubly connected domain $G$ and continuous on the closure of $G$, from its "means" on the boundary $\partial G$ of $G$, provided that an explicit conformal map of $G$ onto the unit disc or an annulus can be found and has a sufficiently smooth extension to $\partial G$ and that $f$ is sufficiently smooth on $\partial D$.

Received by the editors May 1, 1972 and, in revised form, February 5, 1973.

AMS (MOS) subject classifications (1970). Primary 30A82, 30A14.

Key words and phrases. Annulus, mean boundary values, Fourier coefficients, Riemann coefficients, Riemann series, Möbius function, holomorphic function.

(c) American Mathematical Society 1973 
Let $0<r_{0}<1$, and consider the annulus $D=\left\{z: r_{0}<|z|<1\right\}$. For an $\varepsilon>0$, we denote by $A^{1+\varepsilon}(D)$ the class of all functions $f(z)=\sum_{n=-\infty}^{\infty} a_{n} z^{n}$ such that for $n>0, a_{n} \doteq O\left(1 / n^{1+\varepsilon}\right)$ and $a_{-n}=O\left(r_{0}^{n} / n^{1+\varepsilon}\right)$. If $f$ is a function continuous on the boundary $\partial D$ of $D$, we define (cf. [2]) the Riemann coefficients of $f$ by

$$
R_{n}(f)=s_{n}(f)-s_{\infty}(f) \text { and } R_{0 n}(f)=s_{0 n}(f)-s_{0 \infty}(f),
$$

where

and

$$
s_{0 n}(f)=\frac{1}{n} \sum_{k=1}^{n} f\left(r_{0} w_{n}^{k}\right), \quad n=1,2, \cdots
$$

$$
s_{\infty}(f)=\lim _{n \rightarrow \infty} s_{n}(f), \quad s_{0 \infty}(f)=\lim _{n \rightarrow \infty} s_{0 n}(f) .
$$

For all functions $f$ "smooth" on $\partial D$, it is known (cf. [2]) that the Riemann coefficients $R_{n}(f)$ and $R_{0 n}(f)$ have similar asymptotic decay as the Fourier coefficients $a_{n}(f)$ and $a_{0 n}(f)$ respectively, where

$$
a_{n}(f)=\frac{1}{2 \pi} \int_{0}^{2 \pi} f\left(e^{i t}\right) e^{-i n t} d t \text { and } a_{0 n}(f)=\frac{1}{2 \pi} \int_{0}^{2 \pi} f\left(r_{0} e^{i t}\right) e^{-i n t} d t .
$$

It is also known (cf. $[8$, p. 6]) that $f$ is holomorphic in $D$ if and only if $a_{0 n}(f)=a_{n}(f) r_{0}^{n}$ for all $n=0, \pm 1, \cdots$. On the other hand, it is easy to see that for functions $f$ holomorphic in $D, R_{n}(f)$ and $R_{0 n}(f)$ are not related, since there are rational functions $q_{n}$ and $q_{0 n}$ satisfying $R_{m}\left(q_{n}\right)=\delta_{m, n}$, $R_{0 n}\left(q_{m}\right)=0, R_{m}\left(q_{0 n}\right)=0$ and $R_{0 m}\left(q_{0 n}\right)=\delta_{m, n}$ for all $m$ and $n$. However, we will give the relations between $R_{n}(f)$ and $R_{0 n}(f)$ which are necessary and sufficient for functions $f \in A^{1+\varepsilon}(D)$ to be of class $A^{1+\varepsilon}(U)$.

2. Uniqueness, representation and analytical continuability theorems. We first establish the following uniqueness theorem.

THEOREM 1. Let $f \in A^{1+\varepsilon}(D)$ for some $\varepsilon>0$ satisfy

$$
s_{n}(f)=0 \text { and } s_{0 n}(f)=0
$$

for $n=1,2, \cdots$. Then $f$ is the zero function. Furthermore, for each positive integer $n$ there exist two rational functions

$$
q_{n}(z)=\sum_{k=-n}^{n} a_{k} z^{k}, \quad q_{0 n}(z)=\sum_{k=-n}^{n} a_{0 k} z^{k}
$$

with $a_{0}=a_{00}=0$ such that $s_{m}\left(q_{n}\right)=\delta_{m, n}, s_{0 m}\left(q_{n}\right)=0, s_{m}\left(q_{0 n}\right)=0$ and $s_{0 m}\left(q_{0_{n}}\right)=\delta_{n, m}$ for all $m, n=1,2, \cdots$. 
Proof. Since $f$ is holomorphic in $D$, we write $f(z)=\sum_{n=-\infty}^{\infty} a_{n} z^{n}$ with

$$
a_{0}=\frac{1}{2 \pi i} \int_{|z|=1} f(z) \frac{d z}{z}=\lim _{n \rightarrow \infty} s_{n}(f)=0 .
$$

Let $g(z)=\sum_{n=1}^{\infty}\left(a_{n}+a_{-n}\right) z^{n}$. Then $g \in A^{1+\varepsilon}(U)$ and $s_{n}(g)=s_{n}(f)=0$ for all $n=1,2, \cdots$. Hence, we can conclude from a uniqueness theorem in [1] that $a_{n}+a_{-n}=0$ for all $n$. Similarly, we also consider

$$
h(z)=\sum_{n=1}^{\infty}\left(a_{n} r_{0}^{n}+a_{-n} \frac{1}{r_{0}^{n}}\right) z^{n},
$$

and conclude that $s_{n}(h)=s_{0 n}(f), n=1,2, \cdots$, and hence that $a_{n} r_{0}^{n}+$ $a_{-n} r_{0}^{-n}=0$ for all $n$. Since $0<r_{0}<1$, it is clear that $a_{n}=0$ for all $n$.

Next, we prove the existence of $q_{n}$. The proof of the existence of $q_{0 n}$ is similar. Since $s_{m}\left(q_{n}\right)=s_{0 m}\left(q_{n}\right)=0$ for all $m>n$, we need only consider the following two systems of $n$ equations:

$$
\begin{aligned}
& s_{1}\left(q_{n}\right)=\left(a_{1}+a_{-1}\right)+\cdots+\left(a_{n}+a_{-n}\right)=0 \\
& s_{2}\left(q_{n}\right)=\left(a_{2}+a_{-2}\right)+\left(a_{4}+a_{-4}\right)+\cdots=0 \\
& s_{n-1}\left(q_{n}\right)= \\
& \left(a_{n-1}+a_{-(n-1)}\right)=0 \\
& s_{n}\left(q_{n}\right)= \\
& a_{n}+a_{-n}=1 \text {; } \\
& s_{01}\left(q_{n}\right)=\left(a_{1} r_{0}+a_{-1} r_{0}^{-1}\right)+\cdots+\left(a_{n} r_{0}^{n}+a_{-n} r_{0}^{-n}\right)=0 \\
& s_{02}\left(q_{n}\right)=\left(a_{2} r_{0}^{2}+a_{-2} r_{0}^{-2}\right)+\left(a_{4} r_{0}^{4}+a_{-4} r_{0}^{-4}\right)+\cdots=0 \\
& \begin{array}{r}
s_{0, n-1}\left(q_{n}\right)= \\
s_{0 n}\left(q_{n}\right)=
\end{array} \\
& \left(a_{n-1} r_{0}^{n-1}+a_{-(n-1)} r_{0}^{-(n-1)}\right)=0 \\
& a_{n} r_{0}^{n}+a_{-n} r_{0}^{-n}=0 .
\end{aligned}
$$

Since the coefficient matrices for $\left(a_{k}+a_{-k}\right)$ and $\left(a_{k} r_{0}^{k}+a_{-k} r_{0}^{-k}\right)$ are nonsingular, there are unique solutions for $\left(a_{k}+a_{-k}\right)$ and $\left(a_{k} r_{0}^{k}+a_{-k} r_{0}^{-k}\right)$, and hence for $a_{k}$ and $a_{-k}, k=1, \cdots, n$.

To establish our representation theorem, we first obtain explicit formulas for $q_{n}$ and $q_{0 n}$. Let $\mu(n)$ be the Möbius function of $n$ :

$$
\begin{aligned}
\mu(n) & =1, & & \text { if } n=1, \\
& =(-1)^{k}, & & \text { if } n=q_{1} \cdots q_{k}, \\
& =0, & & \text { if } p^{2} \mid n \text { for some } p>1,
\end{aligned}
$$

where $q_{1}, \cdots, q_{k}$ are distinct primes. 
Lemma 1. For each $n=1,2, \cdots$,

$$
q_{n}(z)=\sum_{j \mid n} \frac{\mu(n / j)}{r_{0}^{-j}-r_{0}^{j}}\left\{\left(\frac{z}{r_{0}}\right)^{j}-\left(\frac{z}{r_{0}}\right)^{-j}\right\}
$$

and

$$
q_{0 n}(z)=\sum_{j \mid n} \frac{\mu(n / j)}{r_{0}^{j}-r_{0}^{-j}}\left\{z^{j}-z^{-j}\right\}
$$

Proof. We observe that the means

and

$$
s_{n}\left(\frac{\left(z / r_{0}\right)^{j}-\left(z / r_{0}\right)^{-j}}{r_{0}^{-j}-r_{0}^{j}}\right)= \begin{cases}1, & \text { if } n \mid j, \\ 0, & \text { if } n \nmid j\end{cases}
$$

$$
s_{0 n}\left(\frac{\left(z / r_{0}\right)^{j}-\left(z / r_{0}\right)^{-j}}{r_{0}^{-j}-r_{0}^{j}}\right)=0 \text { for all } n=1,2, \cdots .
$$

Hence, by virtue of Theorem 1, we have

$$
\frac{\left(z / r_{0}\right)^{j}-\left(z / r_{0}\right)^{-j}}{r_{0}^{-j}-r_{0}^{j}}=\sum_{n \mid j} q_{n}(z)
$$

for $j=1,2, \cdots$. We now use the Möbius inversion theorem (cf. [5]) to obtain (2). The proof of (3) is similar.

THeOREM 2. Let $f \in A^{1+\varepsilon}(D)$ for some $\varepsilon>0$. Then the series

$$
\sum_{k=1}^{\infty} R_{k}(f) q_{k}(z)+\sum_{k=1}^{\infty} R_{0 k}(f) q_{0 k}(z)+s_{\infty}(f)
$$

converges uniformly to fon $\bar{D}$ and

$$
\left|f(z)-\sum_{k=1}^{m} R_{k}(f) q_{k}(z)-\sum_{k=1}^{m} R_{0 k}(f) q_{0 k}(z)-s_{\infty 0}(f)\right|=O\left(\frac{1}{m^{\delta}}\right)
$$

uniformly on $\bar{D}$ for any fixed $\delta, 0<\delta<\varepsilon$.

The series (4) is now called the Riemann series of the function $f$ in $D$ (cf. [3]).

PROOF. For $r_{0} \leqq|z| \leqq 1$, we have

$$
\left|q_{k}(z)\right| \leqq \sum_{j \mid k} \frac{1+r_{0}^{2 j}}{1-r_{0}^{2 j}} \leqq \frac{2 d(k)}{1-r_{0}^{2}}
$$

where $d(k)$ denotes the number of divisors of $k$. Using the well-known 
estimate $d(k)=O\left(k^{\varepsilon-\delta}\right)$, where $0<\delta<\varepsilon$ (cf. [5]), and the fact that $R_{k}(f)=$ $O\left(1 / k^{1+\varepsilon}\right)$ and $R_{0 k}(f)=O\left(r_{0}^{k} / k^{1+\varepsilon}\right)$, which follows from the assumptions on $f$ (cf. [2]), we can conclude that the series (4) converges uniformly on $\bar{D}$ to some function $h$, holomorphic in $D$ and continuous on $\bar{D}$. Furthermore, we have

$$
\left|h(z)-\sum_{k=1}^{m} R_{k}(f) q_{k}(z)-\sum_{k=1}^{m} R_{0 k}(f) q_{0 k}(z)-s_{\infty}(f)\right|=O\left(\frac{1}{m^{\delta}}\right)
$$

uniformly on $\bar{D}$. Now, we use Lemma 1 to estimate the Fourier coefficients of $h:$ For $m>0$,

$$
\begin{aligned}
a_{m}(h)=a_{m} & {\left[\sum_{k=1}^{\infty} R_{k}(f) \sum_{j \mid k} \frac{\mu(k / j)}{r_{0}^{-j}-r_{0}^{j}}\left\{\left(z / r_{0}\right)^{j}-\left(z / r_{0}\right)^{-j}\right\}\right.} \\
& \left.\quad+\sum_{k=1}^{\infty} R_{0 k}(f) \sum_{j \mid k} \frac{\mu(k / j)}{r_{0}^{j}-r_{0}^{-j}}\left(z^{j}-z^{-j}\right)+s_{\infty}(f)\right] \\
= & \frac{1}{1-r_{0}^{2 m}} \sum_{k=1}^{\infty} R_{m k}(f) \mu(k)+\frac{r_{0}^{m}}{1-r_{0}^{2 m}} \sum_{k=1}^{\infty} R_{0, m k}(f) \mu(k)=O\left(\frac{1}{m^{1+\varepsilon}}\right) .
\end{aligned}
$$

Similarly, for $m<0$,

$$
\begin{aligned}
a_{m}(h) & =\frac{-r_{0}^{-2 m}}{1-r_{0}^{-2 m}} \sum_{k=1}^{\infty} R_{-m k}(f) \mu(k)+\frac{-r_{0}^{-m}}{1-r_{0}^{-2 m}} \sum_{k=1}^{\infty} R_{0,-m k}(f) \mu(k) \\
& =O\left(r_{0}^{|m|} /|m|^{1+\varepsilon}\right) .
\end{aligned}
$$

Hence, $h \in A^{1+\varepsilon}(D)$ and the means of $h$ are

$$
\begin{aligned}
s_{m}(h) & =s_{m}\left[\sum_{k=1}^{\infty} R_{k}(f) q_{k}+\sum_{k=1}^{\infty} R_{0 k}(f) q_{0 k}+s_{\infty}(f)\right] \\
& =\sum_{k=1}^{\infty} R_{k}(f) \delta_{m, k}+s_{\infty}(f)=R_{m}(f)+s_{\infty}(f)=s_{m}(f),
\end{aligned}
$$

and similarly, $s_{0 m}(h)=R_{0 m}(f)+s_{\infty}(f)=s_{0 m}(f)$, for all $m=1,2, \cdots$. Hence, $f=h$ by Theorem 1 .

For each $n=1,2, \cdots$, let $p_{n}(z)=\sum_{k \mid n} \mu(n / k) z^{k}$ as in [3]. We have

THEOREM 3. Let $f \in A^{1+\varepsilon}(D)$ for some $\varepsilon>0$. Then $f$ is in $A^{1+\varepsilon}(U)$ if and only if for all $m \geqq 1$

$$
R_{0 m}(f)=\sum_{j=1}^{\infty} p_{j}\left(r_{0}^{m}\right) R_{m j}(f) .
$$

Here, it is clear that the series in (5) converges for every $f$ in $A^{1+\varepsilon}(D)$. 
Proof. An easy calculation shows that

$$
\begin{aligned}
R_{0 k}\left(p_{j}\right) & =p_{\alpha}\left(r_{0}^{k}\right) & & \text { if } j=\alpha k \\
& =0 & & \text { if } k \nmid j .
\end{aligned}
$$

In [3], it is proved that if $f \in A^{1+\varepsilon}(U)$ then $f(z)=\sum_{k=1}^{\infty} R_{k}(f) p_{k}(z)+s_{\infty}(f)$ uniformly in $\bar{U}$. Hence, we have, by (6),

$$
R_{0 m}(f)=\sum_{j=1}^{\infty} R_{m j}(f) \sum_{\alpha \mid j} \mu\left(\frac{j}{\alpha}\right) r_{0}^{m \alpha}
$$

which is (5). To prove the converse, we first prove the following identities for all $k$ and $n$ :

Indeed,

$$
\sum_{j \mid k} p_{j}\left(r_{0}^{k n / j}\right) \mu\left(\frac{k}{j}\right)=r_{0}^{n} \mu(k)
$$

$$
\begin{aligned}
\sum_{j \mid k} p_{j}\left(r_{0}^{k n / j}\right) \mu\left(\frac{k}{j}\right) & =\sum_{j \mid k} \mu\left(\frac{k}{j}\right) \sum_{\alpha \mid j} \mu\left(\frac{j}{\alpha}\right) r_{0}^{\alpha k n / j} \\
& =\sum_{j \mid k} \mu\left(\frac{k}{j}\right) \sum_{\alpha \mid j} \mu(\alpha) r_{0}^{k n / \alpha}=\sum_{\alpha \mid k} r_{0}^{k n / \alpha} \mu(\alpha) \sum_{j \mid k k / \alpha)} \mu\left(\frac{k}{j \alpha}\right),
\end{aligned}
$$

so that (7) follows from the identity $\sum_{j \mid n} \mu(j)=\delta_{1, n}$.

From Theorem 2, we have

$$
f(z)=\sum_{j=1}^{\infty} R_{j}(f) q_{j}(z)+\sum_{j=1}^{\infty} R_{0 j}(f) q_{0 j}(z)+s_{\infty}(f)=\sum_{n=-\infty}^{\infty} a_{n} z^{n} .
$$

It is clear that for each $n>0$,

$$
a_{-n}=\sum_{m=1}^{\infty} \frac{\mu(m)}{r_{0}^{n}-r_{0}^{-n}} R_{n m}(f) r_{0}^{n}+\sum_{m=1}^{\infty} \frac{\mu(m) R_{0, m n}(f)}{r_{0}^{n}-r_{0}^{-n}} .
$$

Since $R_{k}(f)=O\left(1 / k^{1+\varepsilon}\right)$, we obtain, by (5) and (7),

$$
\begin{aligned}
\left(r_{0}^{n}-r_{0}^{-n}\right) a_{-n} & =\sum_{m=1}^{\infty} \sum_{j=1}^{\infty} p_{j}\left(r_{0}^{n m}\right) R_{j m n}(f)-\sum_{m=1}^{\infty} r_{0}^{n} R_{n m}(f) \mu(m) \\
& =\sum_{k=1}^{\infty} R_{k n}\left\{\sum_{j \mid k} p_{j}\left(r_{0}^{k n / j}\right) \mu\left(\frac{k}{j}\right)-r_{0}^{n} \mu_{k}\right\}=0 .
\end{aligned}
$$

3. Final remark. The results in this paper are generalizations of those studied in ([1], [3]). Recently, Patil ([6], [7]) has given an explicit representation of an $H^{p}$ function in terms of its boundary values on a small subset $S$ of the unit circle. It is, therefore, also interesting to know whether or not just the arithmetic means of the values of a function $f \in A^{1+\varepsilon}(U)$ at 
points "equally spaced" on $S$ would uniquely determine $f$, and if so, whether or not an "explicit" formula for recapturing $f$ from these means could be given. If $S$ is an arc, some results have been recently obtained in [4].

\section{REFERENCES}

1. C. H. Ching and C. K. Chui, Uniqueness theorems determined by function values at the roots of unity, J. Approximation Theory (to appear).

2. - Asymptotic similarities of Fourier and Riemann coefficients, J. Approximation Theory (to appear).

3. —_. Mean boundary value problems and Riemann series, J. Approximation Theory (to appear).

4. - Analytic functions characterized by their means on an arc, Trans. Amer. Math. Soc. (to appear).

5. G. H. Hardy and E. M. Wright, An introduction to the theory of numbers, 3rd ed., Clarendon Press, Oxford, 1954. MR 16, 673.

6. D. J. Patil, Recapturing $H^{2}$ functions from boundary values on small sets, Notices Amer. Math. Soc. 19 (1972), A-307, Abstract \#72T-B42 (Paper to appear).

7. - Representation of $H^{p}$ functions, Bull. Amer. Math. Soc. 78 (1972), 617-620.

8. D. Sarason, The $H^{p}$ spaces of an annulus, Mem. Amer. Math. Soc. No. 56 (1965). MR 32 \#6256.

Department of Mathematics, Texas A\&M University, College Station, TEXAS 77843 (Current address of C. K. Chui)

Current address (C. H. Ching): Department of Mathematics, University of Melbourne, Parkville, Victoria 3052, Australia 